Kissinger, T., Charrett, T.O.H., James, S.W., Adams, A., Twin, A. and Tatam, R.P. 2015. Simultaneous laser vibrometry on multiple surfaces with a single beam system using range-resolved interferometry. Proceedings of the SPIE, 9525, Optical measurement systems for industrial inspection IX, paper number 952520. DOI: 10.1117/12.2183281

Copyright 2015 Society of Photo-Optical Instrumentation Engineers. One print or electronic copy may be made for personal use only. Systematic reproduction and distribution, duplication of any material in this paper for a fee or for commercial purposes, or modification of the content of the paper are prohibited.

\title{
Simultaneous laser vibrometry on multiple surfaces with a single beam system using range-resolved interferometry
}

\author{
Thomas Kissinger ${ }^{\mathrm{a}}$, Thomas O. H. Charrett ${ }^{\mathrm{a}}$, Stephen W. James ${ }^{\mathrm{a}}$, Alvin Adams ${ }^{\mathrm{b}}$, Andrew Twin ${ }^{\mathrm{b}}$ and \\ Ralph P. Tatam*a \\ ${ }^{a}$ Engineering Photonics, Cranfield University, Cranfield, MK43 0AL, United Kingdom \\ ${ }^{\mathrm{b}}$ Oxford Instruments plc, Tubney Woods, Abingdon, Oxon, OX13 5QX, United Kingdom \\ *r.p.tatam@cranfield.ac.uk
}

\begin{abstract}
A novel range-resolved interferometric signal processing technique that uses sinusoidal optical frequency modulation is applied to multi-surface vibrometry, demonstrating simultaneous optical measurements of vibrations on two surfaces using a single, collimated laser beam, with a minimum permissible distance of $3.5 \mathrm{~cm}$ between surfaces. The current system, using a cost-effective laser diode and a fibre-coupled, downlead insensitive setup, allows an interferometric fringe rate of up to $180 \mathrm{kHz}$ to be resolved with typical displacement noise levels of $8 \mathrm{pm} \mathrm{Hz}^{-0.5}$. In this paper, the system is applied to vibrometry measurements of a table-top cryostat, with concurrent measurements of the optical widow and the sample holder inside. This allows the separation of common-mode vibrations of the whole cryostat from differential vibrations between the window and the sample holder.
\end{abstract}

Keywords: Interferometry, Vibrometry, Displacement Sensing, Range-Resolved Interferometric Signal Processing, LDV, Multi-Surface Vibrometry, Cryogenics, Low temperature

\section{INTRODUCTION}

Interferometric signal processing techniques based on optical frequency modulation use the resulting phase modulation waveform within an interferometer with non-zero optical path difference (OPD) as a carrier in order to extract interferometric phase information. Range-resolved interferometric signal processing based on optical frequency modulation uses the proportionality of the induced phase modulation amplitude to the OPD to additionally demodulate and separate multiple return signals based on their range. In general, the use of optical frequency modulation enables self-referencing interferometric techniques, which offer simple, passive sensor configurations as well as high inherent stability and downlead insensitivity. These have applications in interferometric fibre sensing as well as in displacement sensing or vibrometry. Previous signal processing techniques based on optical frequency modulation include the wellknown pseudoheterodyne technique ${ }^{1,2}$, using linear or sawtooth optical frequency modulation, which has also been employed for range-resolved multiplexing of interferometric fibre-coupled displacement sensors ${ }^{2}$. Sinusoidal optical frequency modulation schemes ${ }^{3-6}$, where the modulation waveform contains only one frequency component and which are thus easier to implement than linear techniques have also been applied to interferometric fibre sensing ${ }^{3-5}$, and to vibrometry in a self-referencing setup on a single surface ${ }^{6}$. Among the sinusoidal techniques, the phase-generated carrier method $^{3}$ is used widely, but is not range-resolved and cannot multiplex interferometers using a single source and photo detector. Employing sinusoidal frequency modulation in conjunction with rectangular gating ${ }^{4,5}$, however, allows interferometric sensors to be multiplexed based on their range ${ }^{5}$. In general, prior linear and sinusoidal range-resolved interferometric techniques ${ }^{2,5}$ required integer ratios between the OPDs of constituent interferometers to be maintained for proper operation. This makes the setup inflexible as well as leaving measurements susceptible to crosstalk from parasitic or unintended signal sources that do not adhere to the integer OPD ratio requirement. In contrast, a recently developed sinusoidal technique ${ }^{7}$, using a smooth window function instead of rectangular gating, permits continuously variable OPD placement of the signal sources, with no apparent penalty in linear operation or crosstalk as long as a minimum OPD separation between the constituent interferometers is observed, greatly increasing the practicality of the approach.

In this work, this novel range-resolved signal processing approach ${ }^{7}$ is applied to multi-surface vibrometry, where a single laser beam is used to achieve simultaneous and independent interferometric measurements of the vibration of two surfaces. In general, in multi-surface vibrometry, the flexibility of the continuously variable OPD placement that the 
technique offers is of particular importance because there is normally no control over the geometry of the objects under test. While multi-surface vibrometry has also recently been performed using optical frequency $\operatorname{combs}^{8}$, the technique presented here allows for a much simpler and cost-effective setup using only a laser diode source with no requirement for an external modulator. In the specific application presented in this paper, the multi-surface vibrometry system is applied to the measurement of the vibration characteristics of a commercial table-top cryostat system ${ }^{9}$ (OptistatDry) produced by Oxford Instruments plc, both during the development phases and for final product characterization. Here, the first signal is provided by the window of the outer vacuum chamber (OVC), while the second signal is reflected off the sample holder inside the chamber, where the sample holder is directly connected to the piston-driven coldhead of the cryostat in order to cool the sample. The coldhead introduces vibrations into the cryostat, in particular to the sample holder through its direct link with the coldhead. This application therefore requires the independent measurements of the movements of the OVC and of the sample holder relative to the optical table. Furthermore, the ability to distinguish between common-mode vibrations of the whole cryostat and differential vibrations between the OVC window and the sample holder inside was of particular interest and great benefit during the development phases, allowing the identification of the origin of the vibrations. The presented scheme allows these measurements to be performed using a single, collimated beam, permitting the simultaneous capture of both signals and allowing numerical extraction of the common-mode and differential vibrations of the surfaces.

\section{SIGNAL PROCESSING}

The novel signal processing approach ${ }^{7}$ employed in this work uses a sinusoidal optical frequency modulation waveform of modulation frequency $f_{\mathrm{m}}$. As illustrated in Fig. 1(a), this waveform has a amplitude $\Delta f$ and is centred around optical frequency $f_{0}$. For an interferometer with non-zero OPD this results in a corresponding sinusoidal phase modulation waveform of phase modulation amplitude $A$, producing a characteristic interferometric signal on the photo detector. For several constituent interferometers the characteristic interferometric signals are superimposed, as visible in the example photo detector signal $U(t)$ in Fig. 1(b). Here, $U(t)$ is calculated for two constituent interferometers with OPDs that result in phase modulation amplitudes of $A_{1}=53 \mathrm{rad}$ and $A_{2}=97 \mathrm{rad}$, which in this example were explicitly chosen to not be integer multiples of each other. The key step in the novel signal processing approach ${ }^{7}$ is the multiplicative application of a smooth window function to the photo detector signal, $U(t)$. In this work, a window function $W(t)$, plotted in Fig. 1(b), containing two individual Gaussian windows that are centred on the two points of inflection of the sinusoidal frequency modulation waveform, is used. Additionally, for comparison with prior work ${ }^{5}$, the rectangular window/gating function $\Pi(t)$ that was used there is also shown in Fig. 1(b).
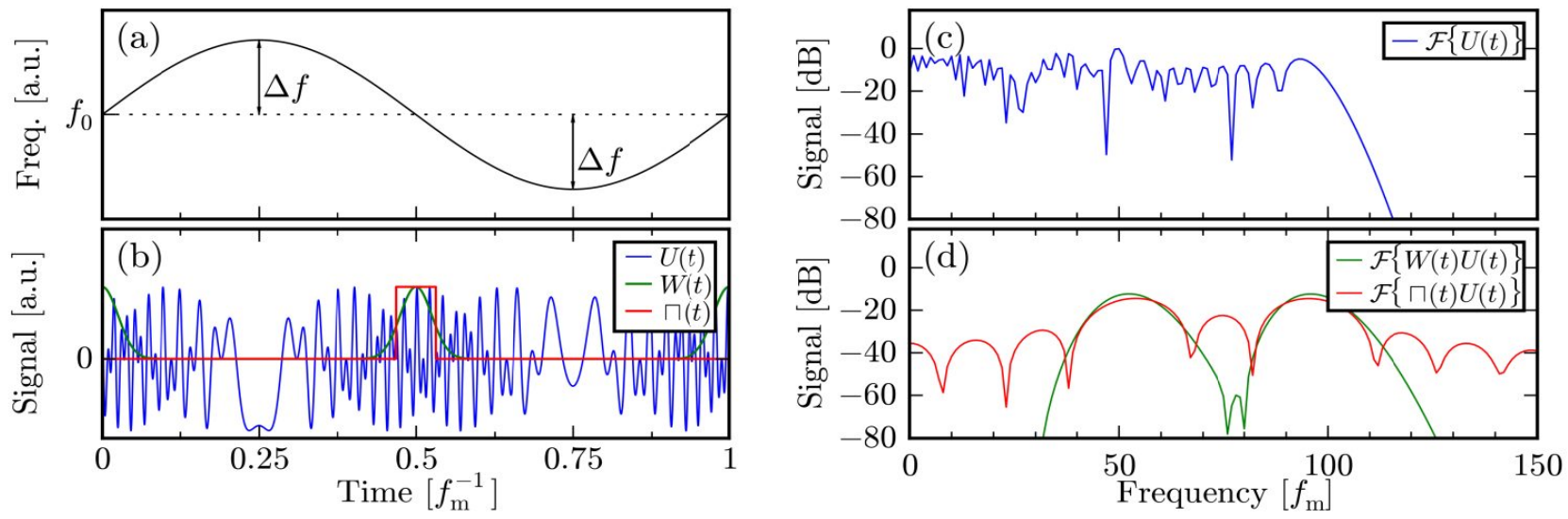

Figure. 1 Illustration of the general principles of the signal processing approach. A sinusoidal optical frequency modulation (a) results in characteristic interferometric signals in the photo detector signal, $U(t)$, that are superimposed for several interferometers, shown in (b) for interferometers with OPD's resulting in phase modulation amplitudes $A_{1}=53$ rad and $A_{2}=97 \mathrm{rad}$, along with the smooth window function $W(t)$ used this approach and the rectangular window function $\Pi(t)$ used in prior $\operatorname{art}^{5}$. (c) and (d) compare the Fourier spectrum of $U(t)$ without (c) and with (d) the application of $W(t)$ or $\Pi(t)$, highlighting the effect of $W(t)$ in separating signals into distinct frequency bands.

The effect of the multiplication of $\mathrm{W}(t)$ becomes obvious when comparing Figs. 1(c) and 1(d). Here, the Fourier spectrum of $U(t)$ on its own can be seen in Fig. 1(c) to be mostly flat, with all Bessel frequency components present. In contrast, the Fourier spectrum of the windowed photo detector signal $W(t) U(t)$ shows a clearly visible separation into 
two distinct peak regions. Here, the peak around $53 f_{\mathrm{m}}$ is found to correspond to the interferometer with phase modulation amplitude $A_{1}$, while the interferometer with phase modulation amplitude $A_{2}$ corresponds to the peak around $97 f_{\mathrm{m}}$. Fig. 1(d) highlights the key principle of the technique, where the multiplicative application of the window function to $U(t)$ in the time domain results in the separation of the signal components of constituent interferometers into distinct frequency bands without introducing sidelobe energy, which could cause crosstalk and non-linear demodulation. This is in stark contrast to the rectangular window function $\Pi(t)$ that was used in prior work ${ }^{5}$, where the corresponding spectrum can be seen to show strong sidelobes and where the avoidance of crosstalk from side lobe components had previously lead to the discussed restriction to integer OPDs, which is absent from this novel approach. A further improvement results from the use of a time-variant complex demodulation carrier ${ }^{7}$, which approximately resembles the expected complex interferometric signal at a given OPD of the constituent interferometer and which can be implemented easily using digital signal processing. In contrast to the use of a fixed frequency carrier, reported in prior work ${ }^{5}$, this reduces the influence of non-idealities arising from the approximation of a section of the sinusoidal phase modulation waveform by a single frequency carrier. Together, both of these measures result in a range-resolved interferometric signal processing technique that is highly linear and flexible, permitting continuously variable OPD placements, and which, furthermore, can be made tolerant to non-idealities resulting from the use of laser injection current modulation, such as associated laser intensity modulation and non-linearities in frequency modulation?

\section{EXPERIMENT}

Fig. 2 shows the optical setup, consisting of a laser diode, fibre-optic circulator and photo detector that are connected using regular single-mode optical fibre, where optical frequency modulation is achieved by sinusoidal modulation of the laser injection current. In this setup, a collimated beam allows the reception of signals from several surfaces. The laser diode used (Eblana Photonics EP1550-NLW-B; centre wavelength: 1551nm, line width: $1 \mathrm{MHz}$ using Profile LDC200 driver) is very cost-effective and all modulation and demodulation is carried out synchronously using low-cost field programmable gate array (FPGA)-based processing (Altera Cyclone IV) at a digital sample rate of $150 \mathrm{MHz}$. In this setup, the interferometric reference is taken from the fibre tip Fresnel reflection, providing an extremely simple, selfreferencing configuration with complete down-lead insensitivity. This also allows a very compact measurement head consisting of only a regular connectorised and packaged fibre optic collimator.

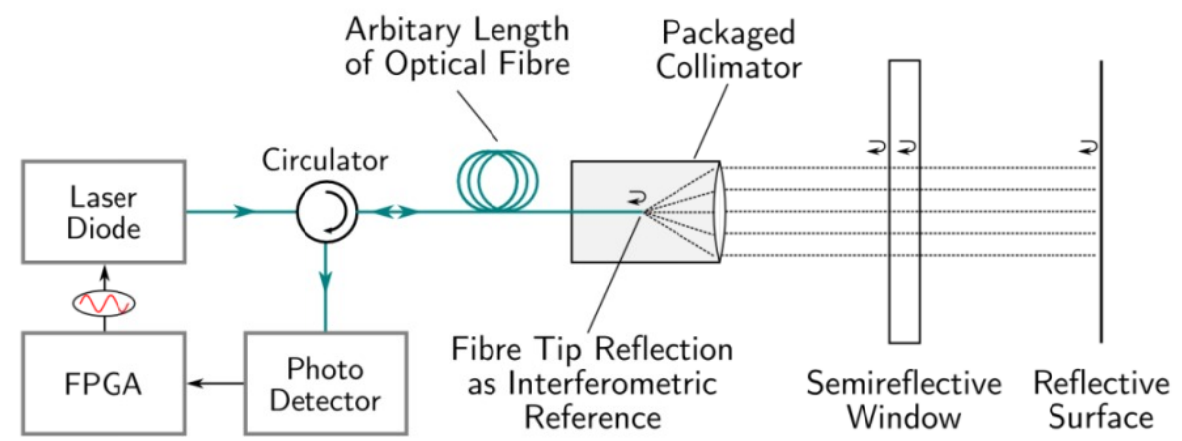

Figure 2. General setup for multi-surface vibrometry. Sinusoidally frequency modulated light from a laser diode is guided by a single-mode optical fibre lead that can be of arbitrary length to a packaged collimator, which then collects light reflected from the semireflective window and the final reflective surface. This light, along with the interferometric reference provided by the fibre tip, reaches the photo detector and the resulting interferometric signals are demodulated by the FPGA.

A picture of the cryostat is shown in Fig. 3(a), while a typical physical measurement arrangement is shown in Fig. 3(b), illustrating a measurement in the X-direction. For the measurement in the Z-direction, the kinematic mount was lowered close to the surface of the optical table and a $45^{\circ}$ prism was inserted under the cryostat to direct the light upwards through the bottom window of the cryostat. In all measurements, the first measurement surface is the window of the OVC, while the second measurement surface is the sample holder inside the cryostat. The measurements shown later were obtained using reflective tape on the sample holder, but successful measurements on other occasions have also been obtained from the unpolished copper surface of the sample holder. In general, in a collimated measurement configuration, return signal powers are lower than for a focused configuration. Also, a collimated beam will result in a higher angular sensitivity than a focused beam. Therefore all measurement surfaces in a setup need to be parallel to each other and the optical axis needs to be aligned using a kinematic mount to achieve perpendicularity to the measurement surfaces. In general, however, the optical configuration is of no relevance to the signal processing, as long as sufficient 
return power is received. For possible future applications in vibrometry of a single surface, a focused configuration, offering higher angular tolerances and providing stronger returned signals, could be used.

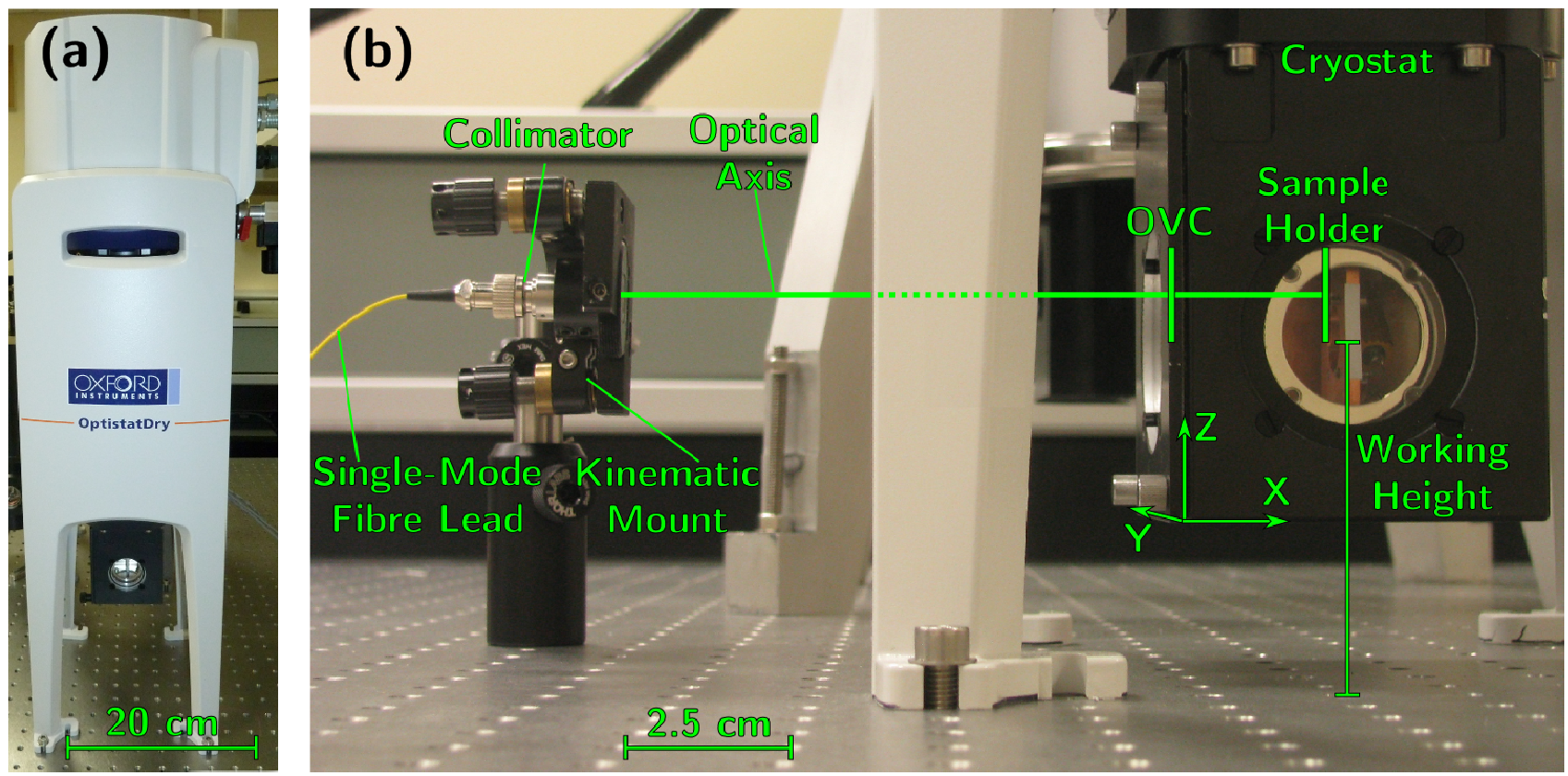

Figure 3. A picture of the whole cryostat is shown in (a), while (b) shows an annotated picture of a typical physical measurement arrangement in the X-direction, where the cryostat was set to different working heights in (a) and (b). In (b), light leaving the single-mode fibre lead and packaged collimator is directed to the OCV window and the sample holder inside the cryostat, with perpendicular alignment to the measurement surfaces achieved using the kinematic mount.

A typical photo detector signal resulting from this arrangement, which also displays some of the undesired influences, such as intensity modulation, is plotted in Fig. 4(a) along with the appropriately delayed window function that is used. After demodulation with the time-variant carrier and the performance of procedures to correct modulation non-idealities ${ }^{7}$ the range dependence of the return signals can be calculated, with the corresponding range dependence shown in Fig. 4(b). Here, the two peaks that originate from the OVC and the sample holder can be identified clearly.
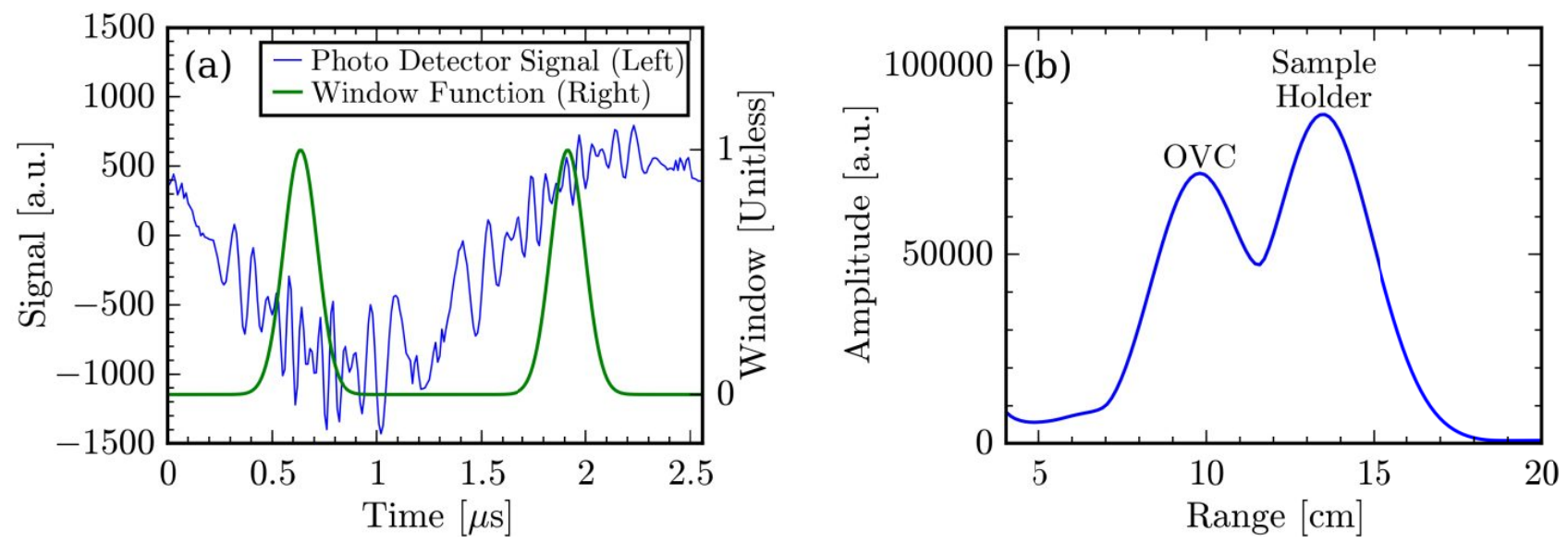

Figure 4. (a) shows a captured photo detector signal over one modulation period along with the appropriately shifted window function on the secondary y-axis. (b) plots the return signals after demodulation with the described time-variant carrier $^{7}$ as a function of range, with the peaks labelled according to the originating signal source.

In the current implementation, the sinusoidal modulation frequency is $f_{\mathrm{m}}=391 \mathrm{kHz}$, allowing an unambiguous interferometric fringe rate of $180 \mathrm{kHz}$, which is slightly lower than the theoretical value of $0.5 f_{\mathrm{m}}$ due to the necessary low-pass filtering in the FPGA. This corresponds to a maximum resolvable velocity of $0.14 \mathrm{~ms}^{-1}$. This could be 
improved through the use of a higher modulation frequency $f_{\mathrm{m}}$ in future implementations. With the current parameters of the implementation, the FWHM width of the peaks in Fig. 4(b) is $3.5 \mathrm{~cm}$. Therefore, the minimally permissible distance between signal sources where no crosstalk will be present, as well as the initial stand-off distance from the fibre-tip, is also approximately $3.5 \mathrm{~cm}$, which is a property of the peak frequency excursion $\Delta f$ achievable by the laser. In this work, the thickness of the window of the OVC is much smaller than the minimum resolvable spatial separation between sources. Therefore the phasor sum of the Fresnel reflections of both window surfaces is effectively measured and taken as the OVC signal, which does not pose any problems as long as the window thickness remains constant.

\section{RESULTS AND DISCUSSION}

Using the described multi-surface vibrometry system, a large body of data has been collected for different working heights and operating temperatures of the cryostat. Fig. 5 shows some measurements at a cryostat temperature of 4 $\mathrm{K}$ and at a working height of the optical windows of $172 \mathrm{~mm}$. These measurements, which were recorded sequentially for each direction, are thought to be representative of the system, although changes in the operating conditions, such as different cryostat temperatures and working heights, result in somewhat different vibration signatures and amplitudes.
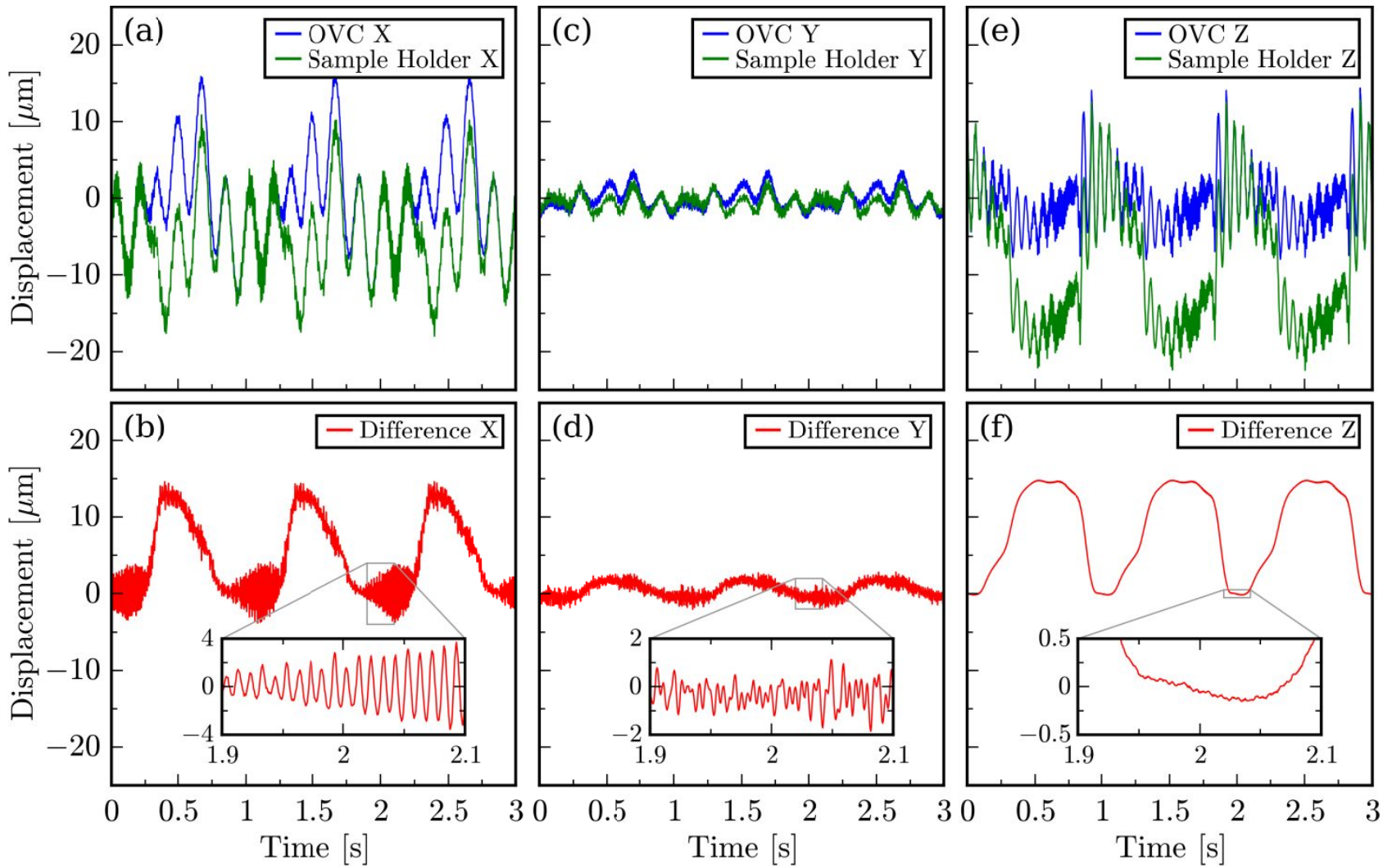

Figure 5. Plot of typical vibration signatures recorded over three seconds for the three measurement directions. Here, the upper panels (a), (c) and (e) directly plot the measured signals for each direction, while the lower panels (b), (d) and (f) show the respective numerically computed difference signal. In each of the lower panels, an inset that plots an enlarged section of the differential vibration signal is drawn, where the y-axis scale in the inset is also increased going from (b) to (f).

For final product characterization of the cryostat, the RMS values are computed, which, for the measurements presented in Fig. 5, results in values of [6.5 $\mu \mathrm{m}, 1.3 \mu \mathrm{m}, 4.1 \mu \mathrm{m}]$ for the $[\mathrm{X}, \mathrm{Y}, \mathrm{Z}]$ vibrations of the $\mathrm{OVC}$, and in values of [5.8 $\mu \mathrm{m}$, $1.0 \mu \mathrm{m}, 8.4 \mu \mathrm{m}]$ for the $[\mathrm{X}, \mathrm{Y}, \mathrm{Z}]$ vibrations of the sample holder. These values are within the RMS specification limit of $10 \mu \mathrm{m}$ for the OptistatDry9. However, during the product development phases, the diagnosis of the origin of the vibrations and their quantification was very important. In all cases plotted in Fig. 5, there is a dominant vibration at $1 \mathrm{~Hz}$ due to the movement of the coldhead piston. However, the OVC and the sample holder show considerable commonmode vibrations, for instance at $\approx 6 \mathrm{~Hz}$ for the $\mathrm{X}$-direction as visible in Fig. 5(a), that are completely absent in the 
corresponding differential signal in Fig. 5(b). In contrast, the differential signal between the OVC and the sample holder in the X-direction shows a vibration at $\approx 95 \mathrm{~Hz}$, which is visible in the inset in Fig. 5(b), and that can thus be attributed to a mechanical resonance of the sample holder structure itself. The most striking result presented here is the differential signal in Fig. 5(f), which shows that in the Z-direction, even in the presence of the $1 \mathrm{~Hz}$ coldhead piston movement, there is a complete absence of any high-frequency differential vibration signals or mechanical resonances of the sample holder. Furthermore, this measurement also demonstrates the complete suppression of common-mode vibrations that are clearly present in Fig. 5(e), but are absent in Fig. 5(f), highlighting the very high measurement quality that can be obtained with the presented multi-surface vibrometry approach.

Noise levels were determined from the differential signal in the Z-direction, recorded when the cryostat cooling system was switched off. As can also be seen in Fig. 5(f), this signal can be expected to be mostly free from environmental noise sources, because these are assumed common to both signal sources and no mechanical resonance appear to exist in the Z-direction. The resulting instantaneous noise standard deviation for the differential signal in Zdirection is $3.4 \mathrm{~nm}$, corresponding to a typical displacement noise level of $\approx 8 \mathrm{pm} \mathrm{Hz}^{-0.5}$. From further measurements it is known that the noise shows a clear range dependency, which indicates that the noise behaviour is dominated by laser phase noise, as would be expected for DFB-type lasers that generally show strong phase noise ${ }^{10}$. Regular commercial vibrometers use low-noise Helium-Neon lasers and can be operated in an OPD-balanced setup. Noise levels in commercial vibrometers ${ }^{11}$ of $0.05 \mathrm{pm} \mathrm{Hz}^{-0.5}$ can thus be achieved, which is considerably lower than the proposed system. However, we believe that there are many applications where the measured displacement noise is not the limiting factor. Instead, the possibility of suppressing environmental noise sources by local referencing of the measurement can be a much more important practical advantage. The ability of this approach to perform common-mode vibration suppression was clearly demonstrated by the example measurements in this paper, where common-mode movements of the cryostat are suppressed to a high degree in the differential signal, as evident in Figs. 5(b), 5(d) and 5(f). Furthermore, the technique allows the suppression of spurious reflections based on their range and also permits the verification of the range of the return signals, which can be useful in practical operation in order to exclude reflections from surfaces that are not of interest. Also, there is no reason why the approach could not be extended to more than two measurement surfaces if required. In general, the low-cost implementation and the simplicity of the fibre-coupled setup means that the system could have applications for permanently installed displacement or vibration sensors, even if only a single surface is evaluated.

\section{CONCLUSION}

A novel range-resolved interferometric signal processing technique has been applied to multi-surface vibrometry. Using a very simple fibre-coupled optical setup with complete downlead insensitivity and with component costs totalling less than $£ 3 \mathrm{k}$, simultaneous measurements of the vacuum window and the sample holder inside a table-top cryostat have been obtained, with the ability to separate signals down to a minimum distance of $3.5 \mathrm{~cm}$. Vibration measurements with typical displacement noise levels of $8 \mathrm{pm} \mathrm{Hz}^{-0.5}$ have been demonstrated and the results highlight the ability of the technique to separate common-mode vibrations of the whole cryostat from differential vibrations between the sample holder and the window. Future applications are envisaged in areas where the cost-effectiveness and robustness of the setup would allow permanently installed vibration or displacement sensors to be used or where the ability for local referencing of the desired signals using a window close to the target would allow the suppression of environmental noise.

\section{ACKNOWLEDGEMENTS}

The authors acknowledge the support of the Engineering and Physical Sciences Research Council (EPSRC) UK, via grant EP/H02252X/1 and EP/G033900/1.

\section{REFERENCES}

[1] Jackson, D. A., Kersey, A. D., Corke, M. and Jones, J. D. C., "Pseudoheterodyne detection scheme for optical interferometers," Electron. Lett. 18, 1081-1083 (1982).

[2] Zheng, J., "Triple-sensor multiplexed frequency-modulated continuous-wave interferometric fiber-optic displacement sensor," Appl. Opt. 46, 2189-2196 (2007).

[3] Dandridge, A., Tveten, A. B., Giallorenzi, T. G., "Homodyne demodulation scheme for fiber optic sensors using phase generated carrier," IEEE J. Quantum Electron. 18, 1647-1653 (1982). 
[4] Kersey, A. D., Lewin, A. C. and Jackson, D. A., "Pseudo-heterodyne detection scheme for the fibre gyroscope," Electron. Lett. 20, 368-370 (1984).

[5] Sakai, I., Youngquist, R. and Parry, G., "Multiplexing of optical fiber sensors using a frequency-modulated source and gated output," J. Lightwave Technol. 5, 932-940 (1987).

[6] Lewin, A. C., Ritter, F. and Siegmund, G., "Laser diode based pseudo-heterodyne interferometer for online process control of wire bonders," Proc. SPIE 4072, 8-18 (2000).

[7] Kissinger, T., Charrett, T. O. H. and Tatam, R. P., "Range-resolved interferometric signal processing using sinusoidal optical frequency modulation," Opt. Express, 23, 9415-9431 (2015).

[8] Boudreau, S. and Genest, J., "Range-resolved vibrometry using a frequency comb in the OSCAT configuration," Opt. Express 22, 8101-8113 (2014).

[9] www.oxford-instruments.com/optistatdry

[10] Bartolo, R. E., Tveten, A. B. and Kirkendall, C. K., "The quest for inexpensive, compact, low phase noise laser sources for fiber optic sensing applications," Proc. SPIE 7503, 750370 (2009).

[11] Johansmann, M., Siegmund, G. and Pineda, M., "Targeting the Limits of Laser Doppler Vibrometry," Proc. IDEMA, 1-12 (2005). 
2015-06

Simultaneous laser vibrometry on

multiple surfaces with a single beam

system using range-resolved interferometry

\section{Kissinger, Thomas}

SPIE

Kissinger, T., Charrett, T.O.H., James, S.W., Adams, A., Twin, A. and Tatam, R.P. 2015.

Simultaneous laser vibrometry on multiple surfaces with a single beam system using

range-resolved interferometry. Proceedings of the SPIE, 9525, Optical measurement systems

for industrial inspection IX, paper number 952520. DOI: 10.1117/12.2183281

https://dspace.lib.cranfield.ac.uk/handle/1826/9684

Downloaded from Cranfield Library Services E-Repository 